AIAA 2001-5042

\title{
MULTI-USER HARDWARE SOLUTIONS TO COMBUSTION SCIENCE ISS RESEARCH
}

\author{
Angel M. Otero \\ National Aeronautics and Space Administration \\ Glenn Research Center \\ Cleveland, Ohio 44135
}

\begin{abstract}
In response to the budget environment and to expand on the International Space Station Fluids and Combustion Facility (FCF) Combustion Integrated Rack (CIR), common hardware approach, the NASA Combustion Science Program shifted focus in 1999 from single investigator PI-specific hardware to multi-user "Minifacilities". These mini-facilities would take the CIR common hardware philosophy to the next level. The approach that was developed re-arranged all the investigations in the program into sub-fields of research. Then common requirements within these subfields were used to develop a common system that would then be complemented by a few PI-specific components. The sub-fields of research selected were droplet combustion, solids and fire safety, and gaseous fuels. From these research areas three mini-facilities have sprung: the Multi-user Droplet Combustion Apparatus (MDCA) for droplet research, Flow Enclosure for Novel Investigations in Combustion of Solids (FEANICS) for solids and fire safety, and the Multi-user Gaseous Fuels Apparatus (MGFA) for gaseous fuels.
\end{abstract}

These mini-facilities will develop common Chamber Insert Assemblies (CIA) and diagnostics for the respective investigators complementing the capability provided by CIR. Presently there are four investigators for MDCA, six for FEANICS, and four for MGFA. The goal of these multi-user facilities is to drive the cost per PI down after the initial development . investment is made. Each of these mini-facilities will become a fixture of future Combustion Science NASA Research Announcements (NRAs), enabling investigators to propose against an existing capability.

$\overline{\text { Copyright }(\mathcal{O} 2001}$ by the American Institute of Aeronautics and Astronautics, Inc. No copyright is asserted in the United States under Title 17, U.S. Code. The U.S. government has a royalty-free license to exercise all rights under the copyright claimed herein for Governmental Purposes. All other rights are reserved by the copyright owner.
Additionally, an investigation is provided the opportunity to enhance the existing capability to bridge the gap between the capability and their specific science requirements.

This multi-user development approach will enable the Combustion Science Program to drive cost per investigation down while drastically reducing the time required to go from selection to space flight.

Introduction

In response to the budget environment in 1999 and to align the Program to better utilize the long term ISS research facility (Combustion Integrated Rack, see figure 1), the Combustion Science Program shifted focus away from individual projects developing customized pieces of hardware for a single Principal Investigator (PI). The new focus drew from our experience gained with the Combustion Module (CM1 project), see figure 2 , in which facility-class hardware was developed to meet the requirements of two totally independent Investigators. This experience was further augmented with the Combustion Module 2 (CM-2) project, see figure 3 . In $\mathrm{CM}-2$, the requirements of three Investigators are satisfied, including a commercial Investigator from the Center for Commercial Application of Combustion in Space (CCACS). These two projects allowed the Combustion Program to develop the expertise to handle "multi-user" hardware developments.

The Mini-facility concept complements the Rack level facilities like the CIR. It takes the next step in searching for common requirements among investigators, developing as much common hardware as
This is a preprint or reprint of a paper intended for presentation at a conference. Because changes may be made belore formal publication, this is made available with the understanding that it will not be cited or reproduced without the permission of the author. 
possible, then concentrating on the necessary $\mathrm{PI}$ specific hardware developments required to accommodate the largest amount of science feasible.

Due to the ever-tightening budget constraints being faced during the ISS assembly phase, it becomes imperative to limit the cost to accomplish science. The mini-facility concept satisfies this criteria, plus it sets

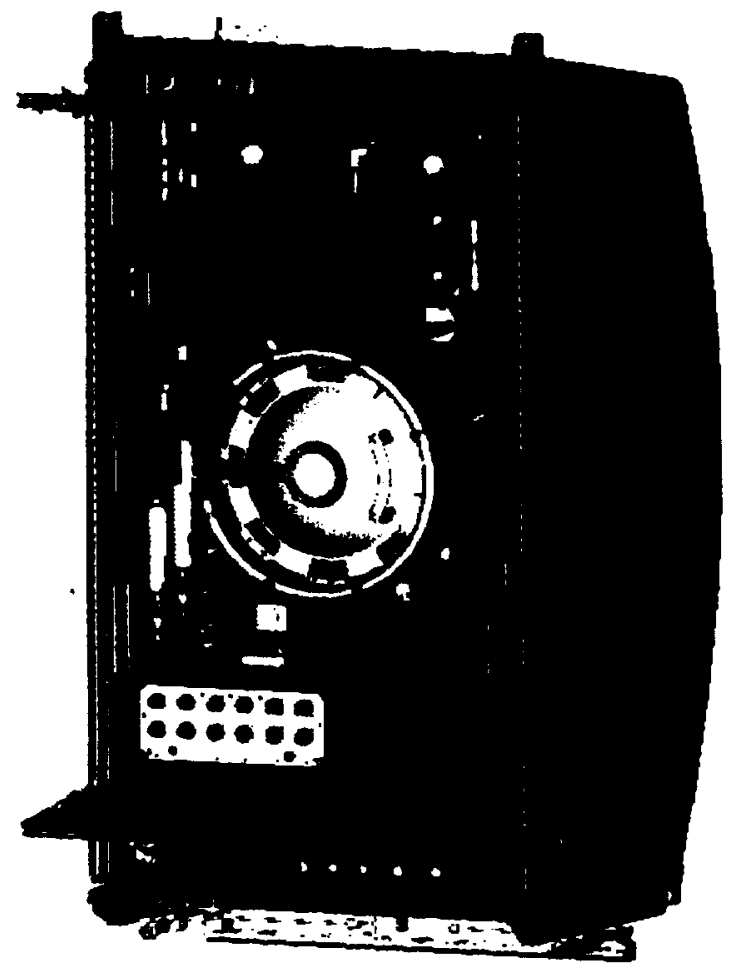

the ground work for much larger savings in the future on hardware development, plus allowing to increase the capability for higher science returns with every NASA Research Announcement (NRA) cycle.

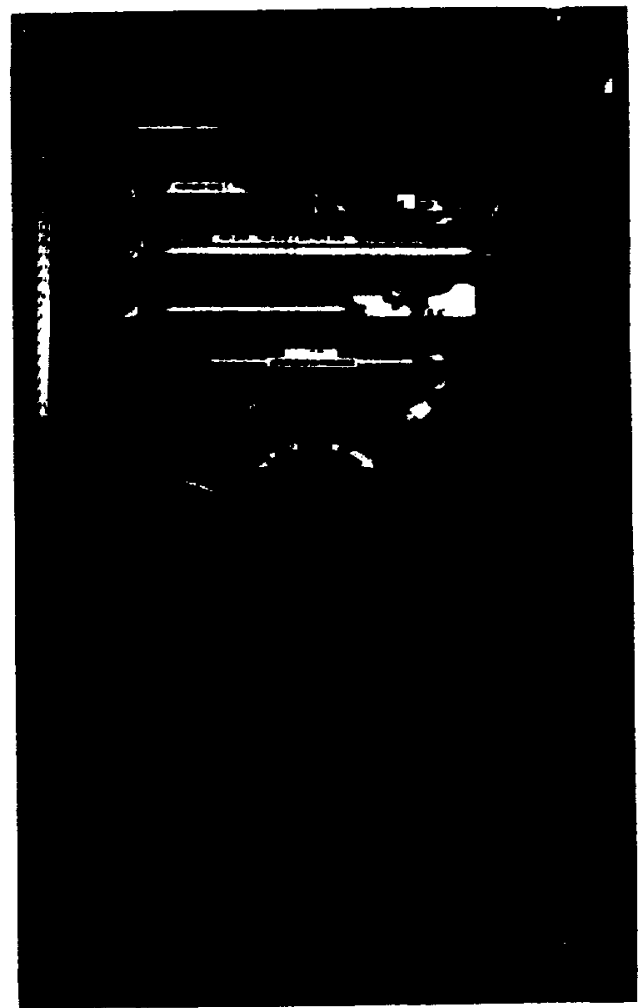

\section{Figure 1}

CIR conceptual view and engineering model 


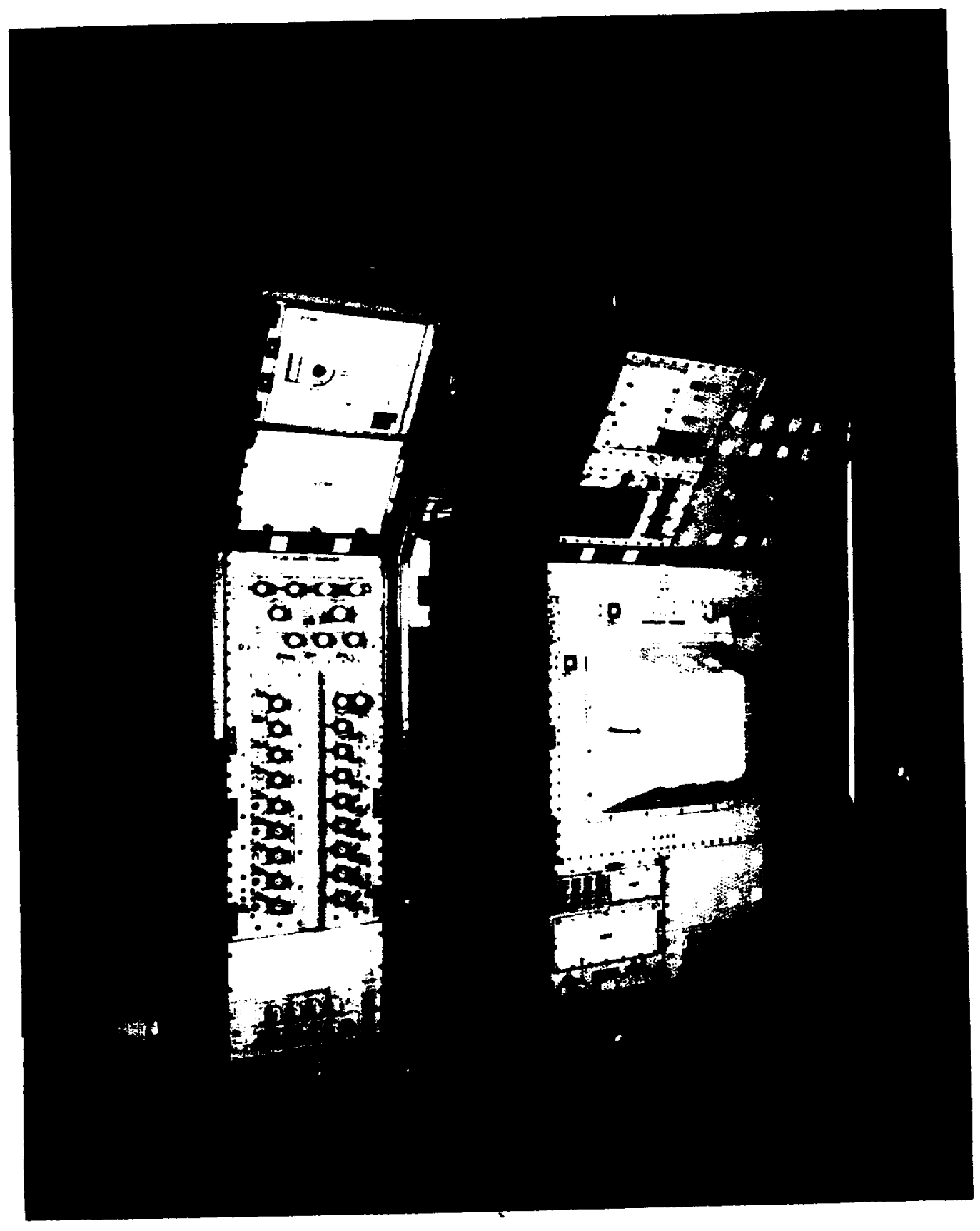

Figure 2

CM-1 single and double rack Spacelab hardware 


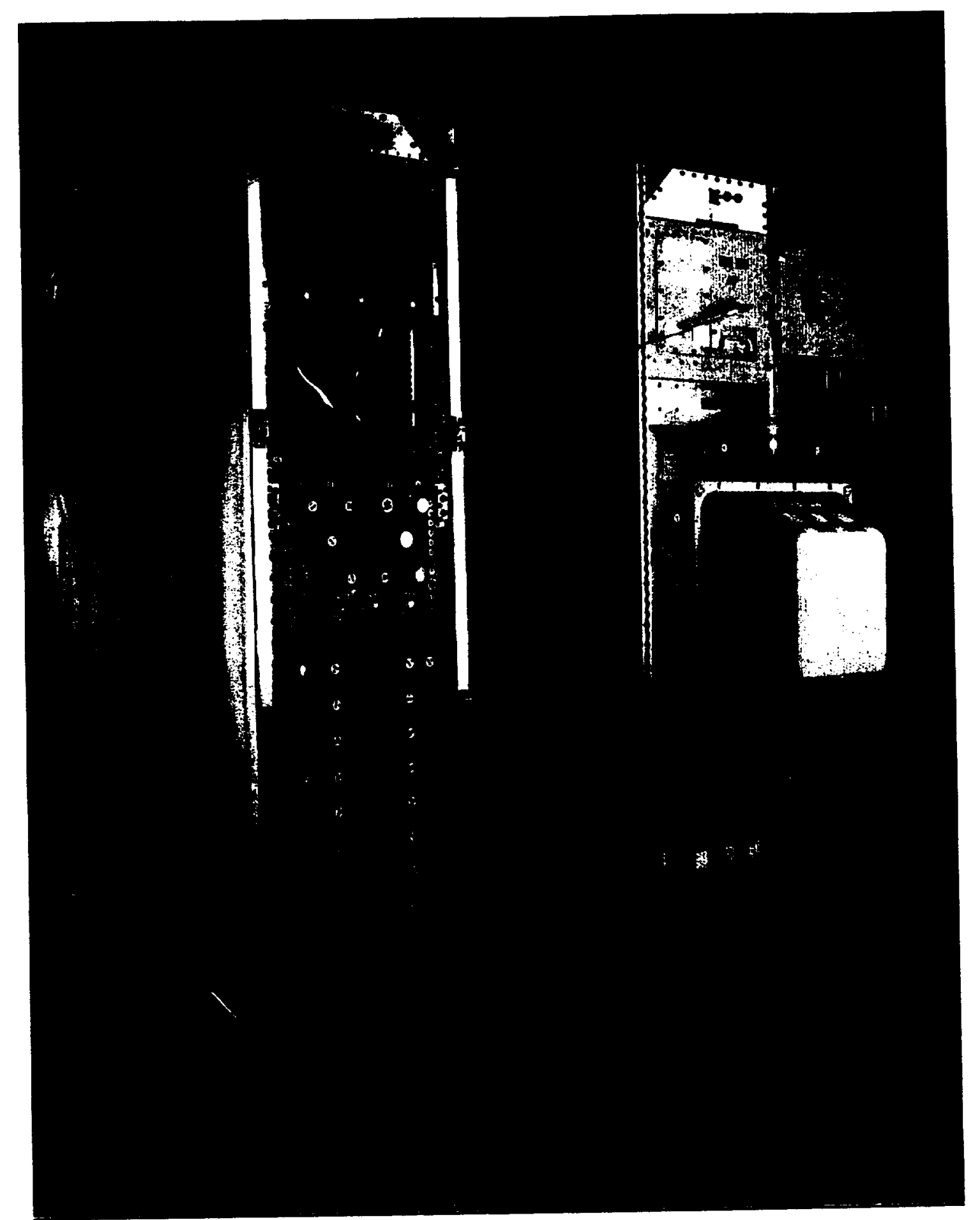

Figure 3

CM-2 SpaceHab hardware 
ISS Era

The advent of the International Space Station (ISS) and the development of the Combustion Integrated Rack (CIR) provided a unique research opportunity to the Combustion Program. The ISS provides long duration access to Microgravity to extend our research beyond the timeframes allowed by the early programs, i.e., Spacelab. The CIR development provided the program incredible savings by developing a combustion chamber for multiple users, thus saving the programs millions of dollars in development cost for individual chambers for individual investigators. Also, the CIR provides diagnostics and gas supply systems that are common to several users, enhancing the cost savings further. (reference 1)

The ISS-CIR combination provides outstanding benefits to the program but also some serious challenges. At the present time, both the ISS and the CIR are been developed simultaneously. The program is faced with the challenge of developing the sub-rack level hardware for the PIs at the same time the carrier and vehicle are in development. Presently, our program has over fifteen Flight investigators planning to use the CIR, see Figure 4. This situation made it imperative that ways were found to reduce development complexities and costs. The solution that made the most sense was to extend the concept of multi-user hardware, like the CIR approach, to the sub-rack level payload. It was noted that most of the present investigators could be logically grouped into similar sub-research categories. Thus the "mini-facility" concept was borm.

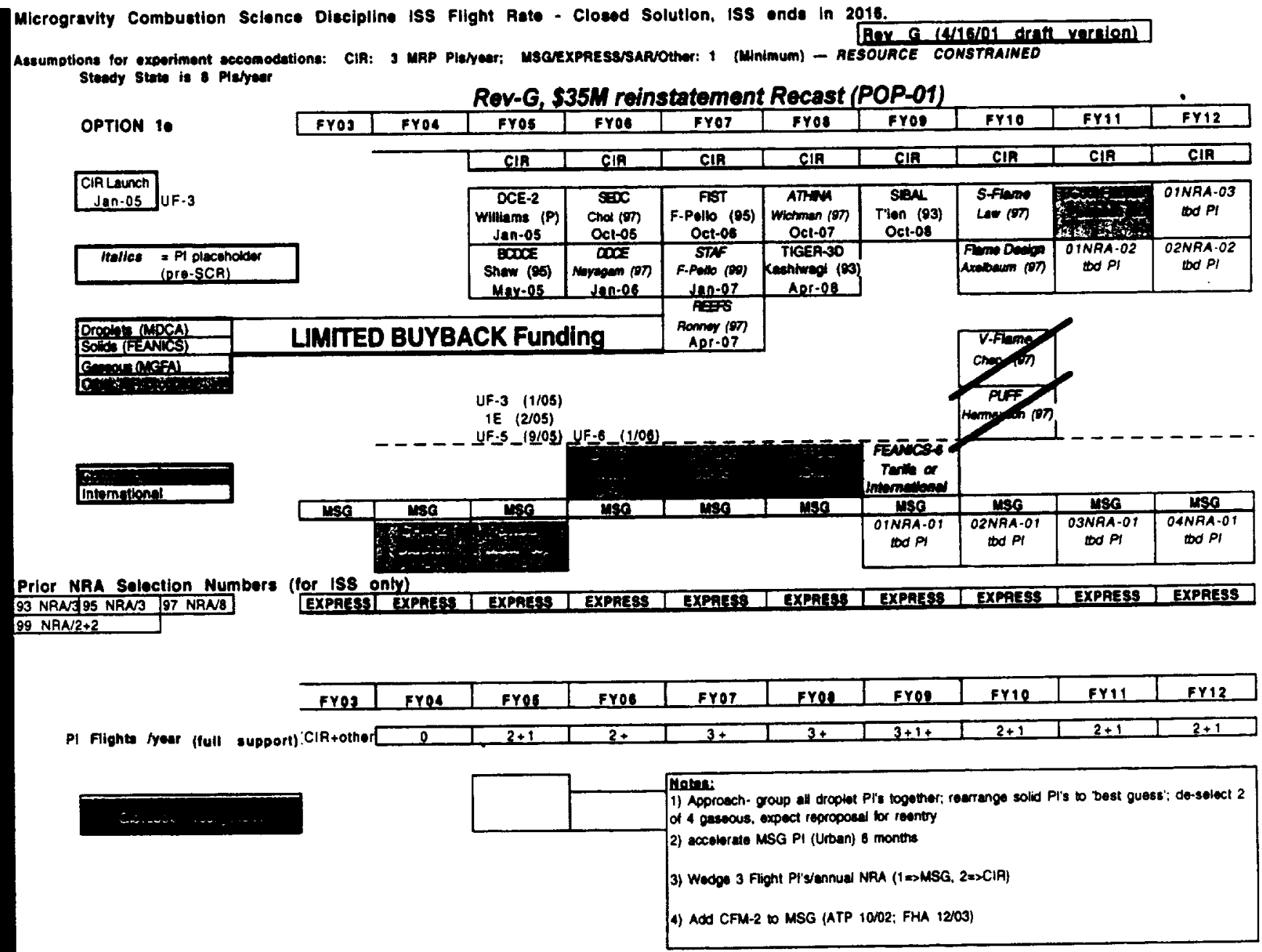

Figure 4

Combustion Program ISS Flight manifest: "Buy-back" Program 


\section{The Concept}

The "mini-facility" concept aligns the hardware development efforts with the sub-fields of research the combustion research community pursues. The Combustion Program research primarily is comprised of four major sub-fields: pre-mixed gases, solids and fire safety, liquids and sprays, and combustion synthesis. Based on the present PI cadre in the Flight program, three of these sub-fields were identified for "mini-facility" hardware developments. They are the Multi-user Droplet Combustion Apparatus (MDCA) for liquids and sprays (reference 2), the Flow Enclosure for Novel Investigations in Combustion of Solids (FEANICS) for solids and fire safety (reference 3 ), and the Multi-user Gaseous Fuels Apparatus (MGFA) for pre-mixed gases (reference 4).

The concept calls for the development of as much commonality as possible. The hardware teams and investigators identified the planned CIR capability that will meet the sub-field requirements. Then a set of common requirements and hardware are identified that will meet the grouped investigators' needs, thus becoming the heart of the "mini-facility". Then small $\mathrm{Pl}$-specific development efforts will be put in place to address any requirements not met with common hardware.

This concept calls for a much closer relationship between the hardware and science teams. Due to the extremely tight budget and schedule environment on ISS, science and engineering requirements need to be clearly identified to avoid cost growth. Since all three components, ISS, CIR, and "mini-facility" are under development simultaneously, and some of the science is not $100 \%$ mature, the hardware and science teams must continue to address the changing environment to ensure the capability required is covered within one of the three components. The concept of compatibility checks has been instituted to ensure that, as the science requirements mature, the capability is still present within one of the three components, or that the implementation of that specific science is delayed until the capability can be put in place.

The real advantage that the "mini-facility concept provides the program is that each one of the "minifacilities" capability will grow with every single PI added to it. After the initial development investment, every new diagnostics developed for a PI becomes part of the inventory of this "mini-facility" for the next NASA Research Announcement (NRA). Therefore every cycle enhances the capability, thus adding to the savings accrued by the program with this approach.

\section{The Future}

The real payoff of the "mini-facility" concept lies in the future. The initial investment on the common hardware will accrue benefit with every NRA cycle. The investigators selected in the future will benefit from the development efforts for earlier investigations ensuring much lower cost per investigation and allowing the future dollars to be used mainly for diagnostics development for these new investigators. In turn, these new diagnostics will become part of the "minifacilities" inventory thus continuing to advance the program's research capabilities.

Also, the "mini-facilities" will not be limited to the ones in work at the present time. As investigators are identified with a sub-field, such as cool flames research, new "mini-facilities may be added to the program. The fact that future dollars will not be encumbered by substantial hardware development costs for every investigation will allow handling an occasional individual investigator that does not fit within a specific available "mini-facility" capability.

\section{Summary}

As we moved into the ISS era with tight budgets and schedules, the development of the "mini-facility" concept allows the Combustion Program the highest flexibility and it provides the opportunity to accomplish the largest amount of research possible during the assembly phase of the ISS. Also, as we look into the future this concept will reduce the hardware development cost thus maximizing the amount of research performed per dollar spent. As this concept matures and is institutionalized it provides the Combustion Program the opportunity to best utilize the resources provided by the ISS-CIR combination in accomplishing its research goals.

Ultimately this concept will reduce the life cycle time of science concept realization to data return while constantly enhancing the research capabilities of the combustion program within the ISS.

\section{References}

1. O'Malley, Terrence; Weiland, K.; The Fluids and Combustion Facility Combustion Integrated Rack: Microgravity Combustion Science on Board the International Space Station, AIAA 2001-4927

2. Myhre, Craig; The Multi-user Droplet Combustion Apparatus, AlAA 2001-5043

3. Frate, David; et al.; FEANICS- A Multi-user Facility for Conducting Solid fuel Combustion Experiments on ISS, ALAA 2001-5079

4. Jones, Jeffrey; Over, A.; Microgravity Gaseous Combustion Flight Hardware, AIAA 2001-5046 